\title{
Desempenho agronômico de cultivares de amendoim na região da Alta Mogiana
}

\begin{abstract}
Guilherme Salis Uitdewilligen $(\mathbb{D}$
Coplana - Cooperativa Agroindustrial, Unidade de Grãos-Sementes, Jaboticabal, SP, gsalis@coplana.com.

Coplana - Cooperativa Agroindustrial, Unidade de $G$ fmlibanore@coplana.com.

Agroindustrial, Unidade de Grãos-Sementes, Jaboticabal, SP,

Programa de Melhoramento do Amendoim - Embrapa, Santo Antônio de Goiás, GO, jair.heuert@embrapa.br.

Programa de Melhoramento kennedybrunno22@gmail.com.

Kennedy Brunno de Brito Martins (D)

Programa de Melhoramento tais.suassuna@embrapa.br.

Taís de Moraes Falleiro Suassuna (iD)

do Amendoim - Embrapa, Santo Antônio de Goiás, GO,
\end{abstract}

\section{RESUMO}

O objetivo do trabalho foi avaliar o desempenho agronômico de cinco cultivares de amendoim, na região da Alta Mogiana, munícipio de Jaboticabal, estado de São Paulo. $O$ experimento foi realizado em campo, em área de rotação com cana-de-açúcar, no sítio Canadá localizado na rodovia SP-333, no ano agrícola 2019/20, com semeadura manual realizada no dia 19 de novembro de 2019. O delineamento experimental foi em blocos casualizados, com quatro repetições. Os tratamentos foram cinco cultivares de amendoim, sendo: BRS 421 OL, BRS 423 OL, BRS 425 OL, IAC OL 3 e IAC 503. Cada parcela experimental era composta por duas linhas de três metros de comprimento, espaçamento de $0,90 \mathrm{~m}$, com intervalo de dois metros entre parcelas e a área útil para avaliação foi de 3,6 $\mathrm{m}^{2}$. O estande médio foi de 14 plantas $\mathrm{m}^{-1}$. Foram avaliadas a massa de 100 grãos $(\mathrm{g})$ e a produtividade de vagens (kg ha-1 e sacas alqueire-1). Houve diferença significativa para as duas variáveis avaliadas. Nas condições experimentais concluiu-se que as cultivares BRS 421 OL, BRS 425 OL, BRS 423 OL e IAC OL 3 obtiveram produtividades acima de $6.500 \mathrm{~kg} \mathrm{ha}^{-1}$ na região da Alta Mogiana.

Palavras-chave: Arachis hypogaea L.; Programa de Melhoramento; Produtividade.

\section{Agronomic performance of peanut cultivars in the Alta Mogiana region}

\begin{abstract}
The objective of the work was to evaluate the agronomic performance of five peanut cultivars, in the Alta Mogiana region, municipality of Jaboticabal, state of São Paulo. The experiment was carried out in the field, in a rotation area with sugar cane, at the Canada site located on the SP333 highway, in the agricultural year 2019/20, with manual seeding carried out on November 19, 2019. The experimental design it was in randomized blocks, with four repetitions. The treatments were five peanut cultivars: BRS 421 OL, BRS 423 OL, BRS 425 OL, IAC OL 3 and IAC 503. Each experimental plot was composed of two lines of three meters in length, with a spacing of $0.90 \mathrm{~m}$, with an interval of two meters between plots and the useful area for
\end{abstract}


evaluation was $3.6 \mathrm{~m}^{2}$. The average stand was 14 plants $\mathrm{m}^{-1}$. The mass of 100 grains $(\mathrm{g})$ and the yield of pods ( $\mathrm{kg} \mathrm{ha}^{-1}$ and bushel bags ${ }^{-1}$ ) were evaluated. There was a significant difference for the two variables evaluated. In the experimental conditions it was concluded that the cultivars BRS 421 OL, BRS 425 OL, BRS 423 OL and IAC OL 3 obtained yields above 6,500 kg ha-1 in the Alta Mogiana region.

Keywords: Arachis hypogaea L.; Improvement Program; Productivity.

\title{
Rendimiento agronómico de los cultivares de maní en la región de Alta Mogiana
}

\begin{abstract}
RESUMEN
El objetivo del trabajo fue evaluar el desempeño agronómico de cinco cultivares de maní, en la región de Alta Mogiana, municipio de Jaboticabal, estado de São Paulo. El experimento se realizó en el campo, en un área de rotación con caña de azúcar, en el sitio de Canadá ubicado en la carretera SP-333, en el año agrícola 2019/20, con siembra manual realizada el 19 de noviembre de 2019. El diseño experimental estaba en bloques al azar, con cuatro repeticiones. Los tratamientos fueron cinco variedades de maní: BRS 421 OL, BRS 423 OL, BRS 425 OL, IAC $O L 3$ e IAC 503. Cada parcela experimental estaba compuesta por dos líneas de tres metros de longitud, con una separación de 0,90 $\mathrm{m}$. con un intervalo de dos metros entre parcelas y el área útil para la evaluación fue de $3.6 \mathrm{~m}^{2}$. El stand promedio fue de 14 plantas $\mathrm{m}^{-1}$. Se evaluó la masa de 100 granos $(\mathrm{g})$ y el rendimiento de las vainas $\left(\mathrm{kg} \mathrm{ha}^{-1}\right.$ y bolsas de bushel-1). Hubo una diferencia significativa para las dos variables evaluadas. En las condiciones experimentales, se concluyó que los cultivares BRS 421 OL, BRS 425 OL, BRS 423 OL e IAC OL 3 obtuvieron rendimientos superiores a $6.500 \mathrm{~kg} \mathrm{ha}^{-1}$ en la región de Alta Mogiana.
\end{abstract}

Palabras clave: Arachis hypogaea L.; Programa de Mejoramiento; Productividad.

\section{Introdução}

Nos últimos anos tem havido aumento na demanda global por amendoim, estimulando o crescimento desta cultura no país. O Brasil ocupa a $12^{a}$ posição no ranking mundial de países produtores, sendo o quinto maior exportador. A região da Alta Mogiana tem um destaque especial, pois a organização de produtores em cooperativas permitiu que pequenos produtores de cana-de-açúcar se tornassem importantes produtores de amendoim (MASSAFERA, 2020). A comercialização do amendoim de alta qualidade requer diversos protocolos de certificação para atender aos exigentes mercados de alimentos do Brasil e no exterior.

Os produtores da Argentina, reconhecida como referência mundial na produção e exportação do amendoim, descrevem o enorme potencial de produção no Brasil, dando ênfase para as áreas disponíveis para expansão da cultura em rotação com a cana-de-açúcar (TODO MANí, 2018). A consolidação da cultura do amendoim no Brasil está ligada de forma direta com a pesquisa e o desenvolvimento de novas cultivares da cultura, que são 
a base da sustentabilidade de toda cadeia produtiva. As parcerias públicasprivadas são fundamentais para acelerar este desenvolvimento (VIZEU, 2019).

Uma característica requerida para essa expansão em áreas de renovação de canaviais está estritamente ligada a precocidade das novas cultivares de amendoim, pois há um tempo interstício de no máximo 130 dias até que ocorra um novo plantio da cana-de-açúcar (BETIOL et al., 2019). O melhoramento genético busca selecionar cultivares que atendam as demandas dos produtores, conferindo alta produtividade, tamanho e formato de grão, resistência às cercosporioses e alto teor de ácido oleico, além de qualidade visual, nutricional, química e sensorial (UITDEWILLIGEN et al., 2017).

Este trabalho objetivou avaliar o desempenho agronômico de cinco cultivares de amendoim, na região da Alta Mogiana, munícipio de Jaboticabal, estado de São Paulo.

\section{Material e métodos}

O experimento foi realizado em campo, em área de rotação com cana-de-açúcar, no sítio Canadá localizado na rodovia SP-333, na região da Alta Mogiana, município de Jaboticabal-SP, no ano agrícola 2019/20 com semeadura manual realizada no dia 19 de novembro de 2019. O delineamento experimental foi em blocos casualizados, com quatro repetições. Os tratamentos foram cinco cultivares: BRS $421 \mathrm{OL}$, BRS 423 OL, BRS $425 \mathrm{OL}$, IAC OL 3 e IAC 503. Cada parcela experimental era composta por duas linhas de três metros de comprimento, espaçamento de $0,90 \mathrm{~m}$, com intervalo de dois metros entre parcelas e a área útil para avaliação foi de $3,6 \mathrm{~m}^{2}$.

Os manejos cultural e fitossanitário foram realizados de acordo com as recomendações para a cultura. $\mathrm{O}$ estande médio foi de 14 plantas $\mathrm{m}^{-1}$. $\mathrm{O}$ arranquio foi realizado no dia 23 de março de 2020, aos 125 dias após a emergência (DAE) e a colheita foi realizada manualmente após dois dias de cura no campo. Foram avaliados mediante contagem e pesagem de grãos e 
vagens: massa de 100 grãos $(\mathrm{g})$ e produtividade de vagens ( $\mathrm{kg} \mathrm{ha}^{-1}$ e sacas alqueire $\left.{ }^{-1}\right)$. Os dados foram submetidos à análise de variância pelo Teste $\mathrm{Fe}$ as médias dos tratamentos foram comparados pelo teste de Scott-Knott, no nível de $5 \%$ de probabilidade, por meio do programa computacional SISVAR 5.6 (FERREIRA, 2019).

\section{Resultados e discussão}

Houve diferenças significativas em todas as variáveis avaliadas (Tabela 1). Observou-se maiores massas de 100 grãos nas cultivares BRS $421 \mathrm{OL}(85,1 \mathrm{~g})$ e IAC 503 (84,9 g). Massa intermediária foi obtida no cultivar BRS $425 \mathrm{OL}(77,4 \mathrm{~g})$. As cultivares BRS $423 \mathrm{OL}(76,5 \mathrm{~g})$ e IAC OL $3(75,8$ $\mathrm{g}$ ), obtiveram as menores massas, demonstrando amplitude granulométrica dos grãos, podendo ser utilizados para diferentes produtos no mercado de amendoim em grãos. Bazanella et al. (2019) também obtiveram massas de 100 grãos semelhantes com as cultivares BRS 423 OL (77,6 g) e IAC OL 3 (76,6 g), em ensaio conduzido em Tupã-SP.

Tabela 1. Massa de 100 grãos (g) e produtividade de vagens ( $\mathrm{kg} \mathrm{ha}^{-1} \mathrm{e}$ sacas alqueire $^{-1}$ ) em função de diferentes cultivares de amendoim na região da Alta Mogiana. Jaboticabal-SP, 2019/20.

\begin{tabular}{cccc}
\hline \multirow{2}{*}{ Cultivares } & Massa de 100 grãos & \multicolumn{2}{c}{ Produtividade de vagens } \\
\cline { 2 - 4 } & $(\mathrm{g})$ & $\left(\mathrm{kg} \mathrm{ha}^{-1}\right)$ & $\left(\right.$ sacas alqueire $\left.^{-1}\right)$ \\
\hline BRS 421 OL & $85,1 \mathrm{a}$ & $7.376,8 \mathrm{a}$ & 714,1 \\
BRS 425 OL & $77,4 \mathrm{~b}$ & $7.199,2 \mathrm{a}$ & 696,9 \\
BRS 423 OL & $76,5 \mathrm{c}$ & $6.761,8 \mathrm{a}$ & 654,5 \\
IAC OL 3 & $75,8 \mathrm{c}$ & $6.664,5 \mathrm{a}$ & 645,1 \\
IAC 503 & $84,9 \mathrm{a}$ & $5.305,4 \mathrm{~b}$ & 513,6 \\
\hline Média & 79,9 & $6.661,5$ & 644,8 \\
\hline CV (\%) & 1,0 & 8,0 & - \\
\hline Pr>Fc & $>0,0001^{*}$ & $0,0005^{\star}$ & - \\
\hline * - significativo a 5\% de probabilidade pelo teste de Scott-Knott; ns - não \\
significativo; C.V. - coeficiente de variação.
\end{tabular}

As cultivares mais produtivas foram a BRS $421 \mathrm{OL}\left(7.376,8 \mathrm{~kg} \mathrm{ha}^{-1}\right)$, BRS $425 \mathrm{OL}\left(7.199,2 \mathrm{~kg} \mathrm{ha}^{-1}\right)$, BRS $423 \mathrm{OL}\left(6.761,8 \mathrm{~kg} \mathrm{ha}^{-1}\right)$ e IAC OL 3 (6.664,5 $\left.\mathrm{kg} \mathrm{ha}^{-1}\right)$, quando comparadas a cultivar IAC 503 que apresentou a menor produtividade $\left(5.305,4 \mathrm{~kg} \mathrm{ha}^{-1}\right)$. A cultivar BRS $421 \mathrm{OL}$ obteve $2.071,4$ $\mathrm{kg} \mathrm{ha}^{-1}$ e 200,5 sacas alqueire-1 a mais que a IAC 503. Betiol et al. (2019), observaram O IAC $503 \mathrm{com}$ produtividade inferior estatisticamente a BRS 
421 OL, BRS 425 OL e BRS 423 OL, nas condições de Ribeirão Bonito-SP. No estudo de Bazanella et al. (2019), a cultivar BRS 425 OL apresentou produtividade de $7.176,5 \mathrm{~kg} \mathrm{ha}^{-1}$, estando entre as maiores produtividades, corroborando ao presente estudo.

\section{Conclusões}

Nas condições experimentais conclui-se que as cultivares BRS 421 OL, BRS $425 \mathrm{OL}$, BRS $423 \mathrm{OL}$ e IAC OL 3 tiveram produtividades acima de $6.500 \mathrm{~kg} \mathrm{ha}^{-1}$ na região da Alta Mogiana.

\section{Agradecimentos}

Os autores agradecem pela cooperação técnica firmada entre Embrapa e Coplana para a realização do presente estudo. Este experimento também está vinculado ao Programa de Melhoramento do Amendoim da Embrapa, no projeto SEG 20.18.01.021.00.

\section{Referências}

BAZANELLA, M. R.; HEUERT, J.; MARTINS, K. B. B.; SUASSUNA, T. M. F.; DEUS, T. J. Desempenho de genótipos de amendoim na Alta Paulista com avaliação de perdas na colheita. In: Anais do Encontro Sobre a Cultura do Amendoim, 16., 2019, Jaboticabal. Anais eletrônicos... Campinas: GALOÁ, 2019. Disponível em: <https://proceedings.science/encontro-amendoim2019/papers/desempenho-de-genotipos-de-amendoim-na-alta-paulista-comavaliacao-de-perdas-na-colheita>. Acesso em: 4 jun. 2020.

BETIOL, R. A. B.; HEUERT, J.; MARTINS, K. B. B.; SUASSUNA, T. M. F. Desempenho de cultivares de amendoim tipo runner na região central do estado de São Paulo, visando a colheita antecipada. In: Anais do Encontro Sobre a Cultura do Amendoim, 16., 2019, Jaboticabal. Anais eletrônicos... Campinas: GALOÁ, 2019. Disponível em: $<$ https://proceedings.science/encontro-amendoim-2019/papers/desempenhode-cultivares-de-amendoim-tipo-runner-na-regiao-central-do-estado-de-saopaulo--visando-a-colheita-antecipada>. Acesso em: 21 abr. 2020.

FERREIRA, D. F. SISVAR: a computer analysis system to fixed effects split plot type designs. Revista Brasileira de Biometria, [S.L.], v. 37, n. 4, p. 529, 20 dez. 2019. http://dx.doi.org/10.28951/rbb.v37i4.450

MASSAFERA, R. Amendoim brasileiro pode ter safra recorde este ano. 2020. Disponível em: <https://www.biodieselbr.com/noticias/materia- 
prima/outras/amendoim-brasileiro-pode-ter-safra-recorde-este-ano-160320>. Acesso em: 20 abr. 2020.

TODO MANÍ. Um gigante adormecido. 2018. Disponível em: <http://www.todomani.com.ar/mani/notas.asp?nid=1046>. Acesso em: 20 abr. 2020.

UITDEWILLIGEN, G. S.; REICH, T. C.; CAPORUSSO, N. B.; SUASSUNA, N. D.; HEUERT, J.; SUASSUNA, T. M. F. Avaliação do desempenho de linhagens de amendoim em Jaboticabal, São Paulo, via modelos mistos. In: Anais do Encontro Sobre a Cultura do Amendoim, 14., 2017, Jaboticabal. Anais eletrônicos... Campinas: GALOÁ, 2017. Disponível em: $<$ https://proceedings.science/encontro-amendoim-2017/papers/avaliacao-dodesempenho-de-linhagens-de-amendoim-em-jaboticabal--sao-paulo--viamodelos-mistos>. Acesso em: 20 abr. 2020.

VIZEU, L. A. Revista Canavieiros. Panorama da cadeira produtiva do amendoim. n. 159, p. 12-13, set. 2019. Disponível em: $<$ https://www.revistacanavieiros.com.br/uploads/pagina/tag/2019/11/e8IGHvh bxTeXEgc9/159-set-baixa.pdf>. Acesso em: 20 abr. 2020. 